\title{
Solar cycle variation of helicity characteristics indicated by SP/Hinode
}

\author{
Juan Hao \& Mei Zhang
}

Key Laboratory of Solar Activity, National Astronomical Observatory, Chinese Academy of Sciences, Datun Road A20, Chaoyang District, Beijing 100012, China

email: haojuan@bao.ac.cn

\begin{abstract}
Helicity characteristics in active regions (ARs) are studied, using so far the most accurate vector magnetic field measurements obtained with SP/Hinode. Our sample includes all ARs observed by SP/Hinode, up to June 2012. The sample is divided into three sub-samples: Cycle 23 (from 2006.11 to 2008.06), Cycle 24a (from 2008.10 to 2010.09) and Cycle 24b (from 2010.10 to 2012.06). We confirm our previous findings that the usual hemispheric helicity sign rule is not obeyed in the descending phase of solar cycle 23 and is obeyed in the ascending phase of solar cycle 24. And we find that the second phase of the solar cycle 24 (Cycle 24b) shows an even stronger evidence of the usual hemispheric helicity sign rule than its first phase (Cycle 24a). It is also found that our previous finding that the strong and weak fields possess the opposite helicity signs is not followed in Cycle 24b, whereas it is weakly followed in Cycle 24a and strongly followed in the descending phase of Cycle 23. This means that this rule also has a solar cycle variation, in addition to the solar cycle variation of the usual hemispheric helicity sign rule, and there is a roughly 2-years time delay between the two.
\end{abstract}

Keywords. Sun: magnetic fields, sunspots, Sun: evolution

\section{Introduction}

Hemispheric helicity sign rule, that is, positive helicity sign in the southern hemisphere and negative helicity sign in the northern hemisphere, has been observed using various instruments in solar cycles 21, 22 and 23 (see Hao \& Zhang 2011 and references therein). There is no argument on the existence of this rule, but there is an argument on whether it is solar cycle dependent or not. Also in our previous works (Zhang 2006; Zhang \& Hao 2011) we found that the strong and weak fields have opposite helicity signs. In this study, we use so far the most accurate vector magnetic field measurements obtained with $\mathrm{SP} /$ Hinode to check these two aspects on helicity characteristics.

\section{The sample and parameters analyzed}

Our sample includes all active regions (ARs) observed with SP/Hinode, up to June 2012. We divide our sample into three sub-samples: 30 ARs in Cycle 23 (from 2006.11 to 2008.06), 34 ARs in Cycle 24a (from 2008.10 to 2010.09) and 75 ARs in Cycle 24b (from 2010.10 to 2012.06). As in Hao \& Zhang (2011), we calculate two helicity parameters. $\alpha_{z}$ is the mean value of local twist, defined as $\alpha_{z}=\overline{(\nabla \times \boldsymbol{B})_{z} / B_{z}} \cdot \alpha_{h c}$ is the normalized mean current helicity density, obtained by $\alpha_{h c}=\frac{\sum(\nabla \times \boldsymbol{B})_{z} B_{z}}{\sum B_{z}^{2}}$. We used two representations of magnetic field measurement. One is related to "flux density", where the longitudinal magnetic field $B_{z}=f \cdot B \cos (\gamma)$ and the transverse magnetic field $B_{t}=\sqrt{f} \cdot B \sin (\gamma)$. The other is the "field strength", where $B_{z}=B \cos (\gamma)$ and $B_{t}=B \sin (\gamma)$. We present the 
first type as $B_{z}^{1}, B_{t}^{1}$ and the second type as $B_{z}^{2}, B_{t}^{2}$. Correspondingly, helicity parameters are presented as $\alpha_{z}^{1}, \alpha_{h c}^{1}$ and $\alpha_{z}^{2}, \alpha_{h c}^{2}$ respectively.

\section{Results}

Figure 1 shows variations of $\alpha_{z}^{1}$ with the latitude. Here $\alpha_{z}^{1}$ is obtained using data points with $\left|B_{z}^{1}\right|>100 G$. The solid lines indicate the results of least-square linear fits. Values of $d \alpha / d \theta$ from the linear fittings are shown at the right top corner of each panel, in the unit of $10^{-9} m^{-1} d e g^{-1}$. We see that for ARs in Cycle 23, $d \alpha / d \theta$ is positive, whereas values of $d \alpha / d \theta$ for Cycle 24a and Cycle 24b are all negative, with the magnitude of $d \alpha / d \theta$ for Cycle 24b larger than that of Cycle 24a. This means that the usual hemispheric helicity sign rule is not obeyed in the descending phase of solar cycle 23 and is obeyed in the ascending phase of solar cycle 24, with the second phase (Cycle 24b) showing a stronger evidence of the usual hemispheric helicity sign rule.
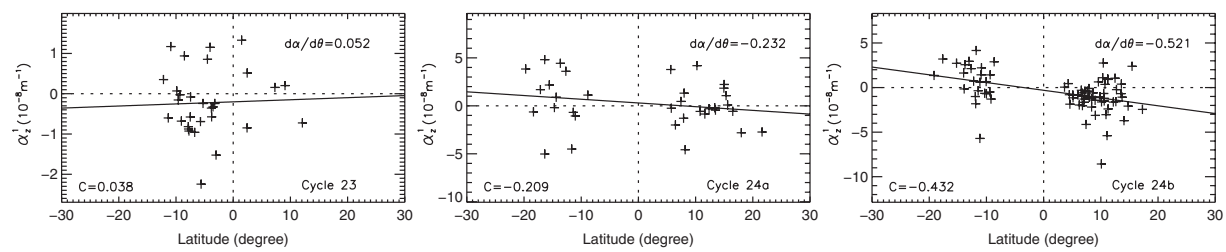

Figure 1. Variations of $\alpha_{z}^{1}$ with the solar latitude for ARs in Cycle 23 (left), in Cycle 24a (middle) and in Cycle 24b (right).

Figure 2 shows the correlation between the weak $\left(100 G<\left|B_{z}^{1}\right|<500 G\right)$ and strong $\left(\left|B_{z}^{1}\right|>1100 G\right)$ fields. Values of the correlation coefficients are shown at the right top corner of each panel. We see that the correlation coefficient between the strong and weak fields is negative in Cycle 23, close to zero for Cycle 24a, and is positive for Cycle 24b. This means that our previous finding that the strong and weak fields possess the opposite helicity sign is not followed in Cycle 24b, whereas it is marginally followed in Cycle 24a and strongly followed in the descending phase of solar cycle 23. This means that this rule also has a solar cycle variation, in addition to the solar cycle variation of the usual hemispheric helicity rule, and there is a roughly 2-years time delay between the two.
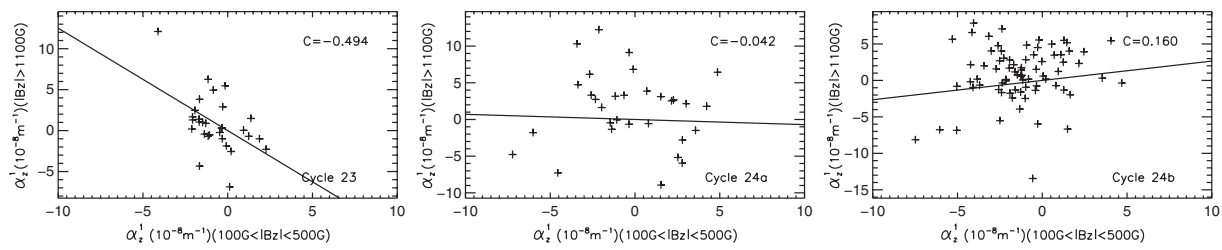

Figure 2. Correlation of $\alpha_{z}^{1}$ between weak and strong fields.

Acknowledgements. We acknowledge supports of the National Natural Science Foundation of China (Grants No. 10921303 and No. 11125314), the National Basic Research Program of MOST (Grant No. 2011CB811401) and the Knowledge Innovation Program of the Chinese Academy of Sciences (Grant No. KJCX2-EW-T07).

\section{References}

Hao, J. \& Zhang, M. 2011, ApJ (Letters), 733, L27

Zhang, M. 2006, ApJ (Letters), 646, L85 